\title{
LES CANARDS CRIMINELS ET LES LIMITES DE LA VIOLENCE DANS LA FRANCE DE LA PREMIÈRE MODERNITÉ
}

\section{Sara Beam}

Armand Colin / Dunod | « Histoire, économie \& société »

2011/2 30e année | pages 15 à 28

ISSN 0752-5702

ISBN 9782200927028

Article disponible en ligne à l'adresse :

http://www.cairn.info/revue-histoire-economie-et-societe-2011-2-page-15.htm

\section{!Pour citer cet article :}

Sara Beam, « Les canards criminels et les limites de la violence dans la France de la première modernité », Histoire, économie \& société 2011/2 (30e année), p. 15-28.

DOI 10.3917/hes.112.0015

Distribution électronique Cairn.info pour Armand Colin / Dunod.

(C) Armand Colin / Dunod. Tous droits réservés pour tous pays.

La reproduction ou représentation de cet article, notamment par photocopie, n'est autorisée que dans les limites des conditions générales d'utilisation du site ou, le cas échéant, des conditions générales de la licence souscrite par votre établissement. Toute autre reproduction ou représentation, en tout ou partie, sous quelque forme et de quelque manière que ce soit, est interdite sauf accord préalable et écrit de l'éditeur, en dehors des cas prévus par la législation en vigueur en France. Il est précisé que son stockage dans une base de données est également interdit. 


\title{
Les canards criminels et les limites de la violence dans la France de la première modernité
}

\author{
par Sara Beam ${ }^{1}$
}

\begin{abstract}
Résumé
Les canards criminels demeurent une source historique relativement négligée bien qu'ils fournissent des informations importantes sur l'évolution des attitudes envers le crime et la violence masculine dans la France de l'époque moderne. Cet article compare ces pamphlets avec les récits de pardons et les mémoires judiciaires, en les abordant à partir de leur utilisation du mot « assassinat ». A la différence de ces derniers genres, les canards condamnaient le criminel et resituaient ses actes dans un contexte de pénitence chrétienne qui est généralement minoré dans les sources que les historiens utilisent habituellement pour comprendre la violence à cette période. Les canards définissent l'assassinat dans un sens bien plus large que le législation de l'époque moderne, et, ce faisant, ils dénonçaient les homicides et les attaques comme des crimes impardonnables. Alors que les récits de pardon tendaient à banaliser la violence masculine, surtout dans les élites, les canards étaient des anti-récits de pardon, qui les jugeaient violemment, et qui insistaient sur le fait que tous les crimes prémédités devaient être pleinement punis par la loi.
\end{abstract}

\section{Abstract}

Les canards criminels are a relatively neglected historical source, that provide important information about changing attitudes to crime and to male violence in early modern France. This study compares these pamphlets with récits de pardon and mémoires judiciaires, with a particular focus on the use of the term "assassination ». Unlike the latter genres, the canards condemn the accused criminal and situate their acts in a Christian penitential context that is normally underplayed in sources relied upon by historians to understand violence in this period. The canards define assassination far more broadly than early modern legislation and, in doing so, censure homicide and assault as unpardonable crimes. Whereas récits de pardon normalize elite male violence, the canards are anti-pardon tales that judge it harshly and insist that any premeditated homicide warrants full punishment under the law. 
Au printemps 1612, dans la ville d'Aix-en-Provence, un jeune home nommé Philibert du Focé demanda à une jeune fille de quinze ans, Perrette de Bon, de l'épouser. Elle refusa en lui disant que sa mère pensait qu'elle était trop jeune pour se marier. Philibert fut surpris et profondément blessé par la réponse de Perrette. Il affirma plus tard qu'il l'aimait depuis presque cinq ans et qu'elle lui avait été promise par son père, qui était décédé depuis. Apparemment, la mère de Perrette, une fois devenue veuve, avait reconsidéré l'engagement nuptial. Le refus de Perrette rendit fou Philibert, une folie, affirma-t-il, exacerbée par le diable qui remplit son âme d'un désir de vengeance et changea son amour passionné en la résolution jalouse de ne souffrir qu'aucun autre homme n'épouse jamais sa Perrette. Fou furieux, Philibert chercha Perrette, lui reprocha de l'avoir repoussé et la poignarda à plusieurs reprises. Quoique grièvement blessée, Perrette s'échappa et s'enfuit à travers les rues en invoquant la justice royale et la justice divine. Elle survécut suffisamment longtemps pour attester que Philibert l'avait attaqué, pour confesser ses péchés et pour mourir d'une belle mort. Pendant ce temps, Philibert, pris de remords, se poignarda mais ne réussit pas à se tuer avant que les magistrats ne s'emparent de lui. Il confessa avoir tué Perrette ; il demanda le pardon des magistrats et que Dieu le punisse. Il obtint ce qu'il avait demandé : il fut condamné à la fois pour le meurtre et pour sa tentative peccamineuse de suicide, à « à estre traisné sur une claye, à faire amende honorable nud en chemise... \& apres mourir honteusement au lieu ou toutes vergongnes sont declarees par le supplice de la corde ${ }^{2} »$.

Cette description triomphante de la capture et de l'exécution de Philibert, inscrite dans une perspective pénitentielle chrétienne, a conduit les érudits à considérer son histoire comme un canard et à insister ses liens avec les autres genres littéraires : les recueils de nouvelles, les traités érudits de morale et les tragédies sanglantes qui étaient aussi populaires à cette époque. Sans rejeter ces références, cet article s'intéresse cependant à l'histoire de Philibert et de Perrette, ainsi qu' aux autres pamphlets courts qui décrivaient ces crimes sensationnels, dans une autre perspective qu'une métaphore pour les questions morales. Il entend, en effet, considérer ces textes dans le cadre d'une réflexion sur la justice criminelle ${ }^{3}$, et examiner la porosité littéraire qui a pu exister entre les canards criminels, les lettres de rémission et les mémoires judiciaires entre les guerres de religion et les premières décennies du XVII ${ }^{\mathrm{e}}$ siècle.

Dans le canard qui raconte cette histoire, publié en 1612, la même année que l'exécution, le crime de Philibert est appelé un « assassinat ». À première vue, appeler un assassinat cette querelle d'amoureux qui tourne mal pourrait paraître étonnant : c'était un crime passionnel et non le meurtre commandité d'une personnalité importante, ce qu'était le sens légal d'un assassinat dans la France au début du XVII ${ }^{\mathrm{e}}$ siècle. Pourquoi ce meurtre, et d'autres qui lui ressemblent, a-t-il été décrit dans les pamphlets de l'époque comme un « assassinat»? Cet article entend montrer que les canards criminels, ces petits pamphlets anonymes publiés sous la forme d'in-octavo bon marché, et qui étaient vendus dans les rues des villes, ont utilisé le terme d'assassinat, à la fois pour choquer les lecteurs et pour avancer des revendications précises concernant l'efficacité et la légitimité de la justice

2. M. Vincent, Discours très véritable de ce qui s'est passé en la ville d'Aix en Provence, le $8^{e}$ iour de juin 1612 entre Philibert Du Focé et Perrette de Bon qui luy avoit esté promise en mariage..., Melun, Pierre de Face, 1612, p. 14-15.

3. Pour une vision bibliographique complète, voir Maurice Lever, Canards sanglants : naissance du fait divers, Paris, Fayard, 1993 ; Jean-Pierre Seguin, L'Information en France avant le périodique : 517 canards imprimés entre 1529 et 1631, Paris, Maisonneuve et Larose, 1964 ; et Frank Greiner (dir.), Fictions narratives en prose de l'âge baroque. Première partie, 1585-1610, Paris, Champion, 2007. 
royale ${ }^{4}$. L'assassinat, à la différence de l'homicide, était un crime irrémissible, à la fois parce qu'il violait la confiance en l'homme et parce qu'il était un péché haineux envers Dieu. Ces pamphlets affirmaient la nécessité de punir ces meurtres par la souffrance physique et par une exécution de la main des officiers royaux. Ce faisant, ils développaient un discours public sur la fonction de la justice du roi et sur sa justification. Plus qu'une propagande baroque de la Contre-Réforme, ces pamphlets ouvrent une fenêtre unique sur l'évolution des attitudes envers la justice royale rétributive et la violence masculine dans la France d'Ancien Régimes .

Pendant cette période, les cours criminelles françaises ont tendu à devenir plus laxistes : entre 1550 et 1650 , moins de suspects ont été torturés pour leur faire avouer leur culpabilité et certains châtiments, comme l'arrachage de la langue ou le bûcher, ont été largement abandonnés ${ }^{6}$. Il est difficile de dire cependant que la violence était en train de disparaître de la société française. Durant les guerres de religion, comme durant la Fronde, la violence entre les personnes, en particulier entre les nobles, est restée très commune ${ }^{7}$. Cette contradiction apparente est bien illustrée par la grande variété des récits criminels qui ont fleuri durant ce siècle. Ce mode d'expression littéraire est devenu populaire pour la première fois durant les années d'incertitude des guerres de religion, lorsque la justice royale échouait, le plus souvent, à contenir à la fois la violence religieuse et les luttes entre les grandes familles nobles. Sa prééminence ne déclina pas avant le milieu du XVII ${ }^{\mathrm{e}}$ siècle, quand l'esthétique plus contrainte du néo-classicisme a modéré l'enthousiasme pour le sensationnel et pour les récits de crimes sanglants, que ce soit sur la scène des théâtres ou dans les livres. Les canards criminels analysés ici ne sont qu'un élément de ce courant littéraire, et ils ne sont pas toujours faciles à distinguer des nouvelles plus longues ou des mémoires judiciaires, produits durant les procès criminels et qui étaient plus directement polémiques ${ }^{8}$. Tous ces genres exprimaient à la fois une fascination et un trouble envers la violence mal contrôlée de la société française.

À la différence des archives qui décrivent et commentent la pratique de la justice criminelle, comme les arrêts, les interrogatoires et les lettres de rémission, les canards

4. Claude Gauvard, «De grace especial » : Crime, État et société à la fin du Moyen Âge, Paris, Publications de la Sorbonne, 1991, 2 vol. ; Robert Descimon, « La royauté française entre féodalité et sacerdoce : roi seigneur ou roi magistrat? » Revue de synthèse, vol. 112, 1991, p. 455-473 ; Malcolm Greenshields, An economy of violence in early modern France : crime and justice in the Haute Auvergne, 1587-1664, University Park, Pennsylvania State, 1994.

5. Roger Chartier, Lectures et lecteurs dans la France d'Ancien régime, Paris, Seuil, 1987 ; Robert W. Scribner, For the Sake of Simple Folk : Popular Propaganda for the German Reformation, Cambridge, Cambridge University Press, 1981 ; Thierry Pech, Conter le crime : droit et littérature sous la Contre-Réforme. Les histoires tragiques, 1559-1644, Paris, Champion, 2000, p. 212-213 ; Maurice Lever, Canards sanglants..., op. cit., p. 14-46.

6. Bernard Schnapper, « La justice criminelle rendue par le Parlement de Paris sous le règne de François $\mathrm{I}^{\mathrm{er}}$ », Revue historique de droit français et étranger, vol. 52, 1974, p. 252-284; Alfred Soman, Sorcellerie et justice criminelle : le Parlement de Paris (16 $-18^{e}$ siècles), Aldershot, Variorum, 1992 ; Robert Muchembled, «Fils de Caïn, enfants de Médée : homicide et infanticide devant le Parlement de Paris (1575-1604) », Annales. Histoires, Sciences sociales, vol. 62, 2007, p. 1063-1094; Jean-Marie Carbasse, Histoire du droit pénal et de la justice criminelle, Paris, Presses Universitaires de France, 2006 ; Benoît Garnot, Crime et justice aux XVII et XVIII ${ }^{e}$ siècles, Paris, Imago, 2000.

7. Stuart Carroll, Blood and Violence in Early Modern France, Oxford, Oxford University Press, 2006 ; Robert Muchembled, L'Invention de l'homme moderne : sensibilités, mours et comportements collectifs sous l'ancien régime, Paris, Fayard, 1988.

8. Denis Crouzet, Les Guerriers de Dieu : la violence au temps des troubles de religion, vers 1525-vers 1610, Seyssel, Champ Vallon, 2 vol., 1990 ; Pascal Champain, Le roman français du XVII e siècle, un genre en question, Paris, L'Harmattan, 2007 ; Théâtre de la cruauté et récits sanglants en France : XVI ${ }^{e}$-XVII ${ }^{e}$ siècle, Christian Biet (dir.), Paris, R. Laffont, 2006 ; Thierry Pech, Conter le crime..., op. cit. 
criminels sont rarement consultés par les historiens de la France moderne ${ }^{9}$. Alors que les historiens de l'Angleterre ont fait bon usage des pamphlets, des ballades et des discours d'échafaud pour explorer les relations violentes entre les sexes, et l'eschatologie protestante et les mentalités populaires ${ }^{10}$, les historiens français semblent effrayés à l'idée d'utiliser ces textes aux dimensions ouvertement fictionnelles. Leurs hésitations sont justifiées par les histoires improbables que racontent certains de ces canards - qui semblent plus proches de « légendes urbaines » actuelles que de récits historiques. L'histoire de Philibert et de Perrette, pour ne parler que d'elle, ressemble de manière frappante à un autre pamphlet, publié six ans auparavant et qui évoquait déjà un amant poignardant fatalement sa fiancée ${ }^{11}$. Cette tendance des canards à se copier les uns les autres jette un doute sur une affirmation récurrente de leurs auteurs, celle qu'ils rapportaient les événements évoqués d'une manière véridique. Quoique d'autres sources puissent parfois corroborer leurs histoires ${ }^{12}$, le vrai intérêt du genre réside peut-être justement dans les fictions qu'il générait.

Les narrations à l'œuvre dans ces pamphlets sont souvent contradictoires avec d'autres documents, tout aussi fictifs, que les historiens consultent régulièrement lorsqu'ils écrivent l'histoire de la justice criminelle : les lettres de rémission et les mémoires judiciaires, qui sont des textes polémiques cherchant à exonérer de ses actions au moins une des parties en présence dans un crime ${ }^{13}$. Par contraste, les canards criminels, qui étaient généralement écrits après l'exécution du principal protagoniste, s'intéressaient surtout à la punition, avec en vue, dans la plupart des cas, la possible réconciliation du criminel avec Dieu. Ces pamphlets, dont des centaines d'exemplaires ont été publiés entre les années 1570 et les années 1630, racontent donc une histoire qui leur est propre et qui complète celle des autres sources historiques. Les contes qu'ils véhiculent, faits d'horribles trahisons, d'interventions providentielles, et d'âmes justifiées par une violence pénitentielle, offrent une vision alternative à l'historien qui cherche à comprendre la complexité et la variation des attitudes envers l'homicide qui coexistent durant les guerres de religion et les décennies qui ont suivi.

L'utilisation des termes « assassin » et « assassinat » dans les canards est une nouveauté car il élargit d'une manière significative la définition légale du concept qui avait cours à l'époque. «Assassin » et « assassinat » étaient, de toute façon, des mots relativement récents dans la France du XVI ${ }^{\mathrm{e}}$ siècle $^{14}$. Ils étaient le plus souvent utilisés dans un contexte

9. Natalie Zemon Davis, Fiction in the Archives : Pardon Tales and their Tellers in Sixteenth-Century France, Stanford, Stanford University Press, 1987, p. 64-65. Le livre de Dorothea Nolde, Gattenmord : Macht und Gewalt in der Frühneuzeitlichen Ehe, Köln, Böhlau, 2003, est une exception.

10. Frances E. Dolan, Dangerous familiars : representations of domestic crime in England, 1550-1700, Ithaca, Cornell University Press, 1984 ; Malcolm Gaskill, Crimes and mentalities in early modern England, Cambridge, Cambridge University Press, 2002 ; Andrea McKenzie, Tyburn's martyrs : execution in England, 1675-1775, London, Hambledon, 2007 ; Peter Lake et Michael C. Questier, The Anti-Christ's lewd hat : Protestants, Papists and players in Post-Reformation England, New Haven, Yale University Press, 2002.

11. N. Serpolet et B. Farge, Discours tres-veritable de ce qui s'est passé en la ville de Molins en Bourbonnois, le huictiesme jour de fevrier 1606. entre Fiacre de saint Germain, et Marie Barbotain, qui luy avoit esté promise en mariage...

12. Pascal Bastien, L'exécution publique à Paris au XVIII siècle : une histoire des rituels judiciaires, Seyssel, Champ Vallon, 2006, p. 216-17 ; Maurice Lever, Canards sanglants..., op. cit., p. 100-101.

13. Natalie Davis, Fiction..., op. cit. ; Sarah C. Maza, Private lives and public affairs : the causes célèbres of prerevolutionary France, Berkeley, University of California Press, 1993 ; Malcolm Gaskill, Crime and Mentalities..., op. cit. ; Roger Chartier (dir.), Les usages de l'imprimé : (XVe-XIXe siècle), Paris, Fayard, 1987 ; Pierre Bourdieu, Esquisse d'une théorie de la pratique, Genève, Droz, 1972.

14. Claude Gauvard, « La violence commanditée : la criminalisation des tueurs à gages aux derniers siècles du Moyen Âge », Annales. Histoire, Sciences Sociales, vol. 62, 2007, p. 1005-1029 ; Dictionnaire francois latin 
juridique et avec un sens relativement étroit. S'appuyant explicitement sur la loi romaine et sur les développements récents des pratiques juridiques françaises, les juristes de la Renaissance et du début du XVII ${ }^{\mathrm{e}}$ siècle, comme André Tiraqueau, Claude Le Brun de la Rochette, et Adam Théveneau ont défini un assassin comme un tueur à gages et ils ont avancé que celui qui commettait un assassinat devait toujours être condamné à mort. Pour ces experts en droit, un meurtre devait être considéré comme un assassinat s'il y avait eu un paiement et en raison de l'intention de l'agresseur sans tenir compte du fait que l'acte s'était ou non conclu par un décès ${ }^{15}$. Il semble que ces juristes étaient principalement concernés par le fait de mieux définir la violence meurtrière des domestiques qui vivaient dans les maisonnées des grands nobles comme illégale et sujette à la justice royale. La législation française du XVI $\mathrm{I}^{\mathrm{e}}$ siècle a suivi la même logique : un édit royal de 1547 stipule qu'un « assassinement » avéré méritait le supplice de la roue, ce qui était, en principe, réservé aux crimes haineux comme le banditisme ou les crimes de lèse-majesté16.

De la même manière, l'édit de Blois, en 1579, définit les assassins comme « ceux qui pour prix d'argent, ou autrement, se loüént pour tuer, outrager, exceder aucuns ». La législation spécifie que ceux qui sont convaincus de ce crime ne peuvent demander « aucune grâce ou rémission ${ }^{17}$ ». En 1621, encore, le juriste Laurent Bouchel définit les « assassinats » comme des meurtres au cours desquels la personne attaquée est un membre de la haute noblesse qui tombe dans une embuscade tendue par des soldats professionnels ${ }^{18}$. Il est donc clair que pour les textes juridiques de l'époque, l'assassinat implique la préméditation, le professionnalisme des attaquants et le fait que les victimes soient des officiers de l'État et/ou des sujets éminents. La loi française du XVI ${ }^{\mathrm{e}}$ siècle considérait donc l'assassinat comme un crime très étroitement défini qui requérait l'exécution du criminel.

Ainsi défini, l'assassinat était donc une forme spécifique et particulièrement répréhensible d'homicide, le terme général pour désigner les crimes au cours desquels une personne causait la mort d'une autre. Quoique les homicides soient aussi un crime capital dans la France d'Ancien Régime, il était possible en pratique pour les accusés de demander un pardon sous la forme d'une lettre de rémission, rédigée par la Chancellerie au nom du roi. Pour prendre effet, ces lettres devaient être enregistrées par la cour qui menait la procédure. Si elles étaient obtenues, les lettres épargnaient la vie de l'accusé qui devait habituellement payer une amende à la famille de la victime et parfois aussi passer plusieurs années aux galères ${ }^{19}$. Comme l'ont montré bien des historiens, la facilité avec laquelle les accusés de meurtres obtenaient des lettres de rémission révèle une société dans laquelle la violence mâle, en particulier la violence nobiliaire, pouvait être justifiée et pardonnée

contenant les motz et manières de parler francois, tournez en latin, Paris, R. Estienne, 1539 ; Dictionnaire francois-latin : auquel les mots françois, avec les manieres d'user d'iceulx, sont tournez en latin..., Paris, G. de Hus, 1573.

15. Claude Le Brun de la Rochette, Le proces civil et criminel, divisé en cinq livres..., Rouen, Pierre Calles, 1611, t. 2, p. 68-69 ; Adam Théveneau, Commentaire de M. Adam Theveneau,... sur les ordonnances... tant en matière bénéficialle que civile et criminelle..., Paris, J. Guignard, 1629, p. 912-914 ; André Tiraqueau, Le «De poenis temperandis » de Tiraqueau, introd., trad. et notes par André Laingui, Paris, Économica, 1986, p. 208.

16. Recueil général des anciennes lois françaises, depuis l'an 420 jusqu'à la Révolution de 1789..., Paris, Plon, 1821, t. 13, p. 26-28.

17. La législation pénale de la France $d u X V I^{e}$ au XIX $X^{e}$ siècle : textes principaux, réunis par Yves Jeanclos, Paris, Presses Universitaires de France, 1996, p. 21.

18. Laurens Bouchel, La ivstice criminelle de la France signalee des exemples les plus notables, depuis l'establissement de ceste Monarchie iusques à present, Paris, Jean Petit-Pas, 1621, p. 430-475.

19. Bien que le Parlement de Paris ait rejeté peu de lettres de rémission, il était aussi rare qu'un pardon complet soit accordé. Voir, par exemple, AN, X2a 119, 8 mai 1556 ; AN, X2a 144, f. 175r, 9 mars 1584 ; AN, X2a 144, f. 220v, 17 mars 1584. 
pourvu qu'un certain nombre de critères soient réunis, en particulier que le crime ait été commis sous le coup de la «colère chaude » ou de la légitime défense ${ }^{20}$. Dans ces récits de pardon, l'intention criminelle du meurtrier est minimisée : le crime est présenté comme accidentel ou justifié par des circonstances atténuantes inévitables. En conséquence, un procès commencé pour homicide avait plus de chance de s'achever par un pardon que par une exécution.

Parmi les premiers pamphlets qui ont employé le terme « assassin » figurent ceux qui racontaient les homicides de rois ou de grands nobles : le meurtre des frères de Guise en 1588 , celui d'Henri III en 1589 et les différentes tentatives contre la vie d'Henri IV, jusqu'à celle réussie par Ravaillac en 1610, ont tous été racontés dans le moindre détail dans des pamphlets qui étaient destinés non seulement à condamner ces actes criminels particulièrement outrageants, mais aussi à faire l'apologie de positions politiques très spécifiques. Les criminels étaient souvent présentés comme des fanatiques religieux voués à la destruction physique et spirituelle de leur victime, un usage qui s'appuyait sur les débuts étymologiques du mot « assassin » dans les langues européennes. Le mot a été, en effet, introduit dans la langue française après les croisades à partir d'un dialecte italien ; un assassin était alors défini comme un Musulman consommateur de haschich et armé de pied en cap en vue de la destruction de la chrétienté ${ }^{21}$. Ces meurtriers notoires, et la littérature pamphlétaire qu'ils ont générée, ont donc aidé à créer une nouvelle conscience publique de l'« assassinat », que se sont ensuite appropriés les auteurs de pamphlets cherchant à publiciser d'autres meurtres, plus ordinaires, entre des sujets du roi de France.

Les canards qui présentent comme des assassinats les meurtres qui résultent de querelles personnelles ou de disputes quotidiennes des Français et des Françaises sont encore plus étonnants. Le genre de querelle amoureuse qui avait poussé Philibert au crime est juste un début : les canards se sont en effet mis à parler d'assassinats pour décrire à peu près tout, du meurtre d'un mari par l'amant de sa femme ou de l'embuscade tendue à un homme de loi par un prétendu ami, jusqu'au viol et au meurtre d'une jeune fille par un officier de l'armée, ou au massacre d'une veuve innocente pour lui voler un coffre rempli d'objets précieux dissimulés dans son grenier ${ }^{22}$. Un pamphlet de 1618 décrit même comme un « assassinat » le meurtre par méprise d'un fils par ses parents ${ }^{23}$. Le fils revenait dans sa ville natale de Nîmes, où ses parents tenaient un hôtel, après une dizaine d'années passées à l'armée. Le pauvre hôtelier et sa femme, ne réalisant pas que le jeune homme, en apparence riche, était leur fils (car il souhaitait leur faire la surprise de son retour et avait seulement dit à sa sœur qu'il rentrait), le tuèrent de sang-froid afin de lui voler les pièces dont son coffre était

20. Natalie Davis, Fiction..., op. cit. ; Stuart Carroll, Blood..., op. cit. ; Claude Gauvard, «De grace especial »..., op. cit. ; Benoît Garnot, Questions de justice : 1667-1789, Paris, Belin, 2006 ; Michel Nassiet, «Vengeance in sixteenth-century France », dans Stuart Carroll (dir.), Cultures of violence : interpersonal violence in historical perspective, New York, Palgrave Macmillan, 2007, p. 117-28.

21. Trésor de la langue française, mis en ligne le 5 mars 2002. URL : http://atilf.atilf.fr/dendien/scripts/tlfiv5/ affart.exe?19;s=250722630;?b=0. Consulté le 16 septembre 2010. Jean Nicot, Thresor de la langue francoyse, tant ancienne que moderne, Paris, David Douceur, 1606, mis en ligne 2001. URL: http://portail.atilf.fr/cgibin/ getobject_?p.0:51./var/artfla/dicos/TLF_NICOT/IMAGE/. Consulté le 15 septembre 2010.

22. Histoire prodigieuse de l'assassinat commis en la personne d'un jeune advocat. Advenue dans Tholose, par la conspiration de sa femme..., Lyon, Leon Savine, 1609 ; Cruaute plus que barbare du massacre commis en la ville de Mets, és personnes de Mangeon vefve du feu André Crepine..., Paris, 1610 ; Les pitoyables et funestes regrets de Marguerite d'Auge sur l'assassin commis par I. Iumeau, sur Claude Antoine son mary..., Lyon, Fleury Durand, 1600 ; Exemplaire punition du violement et assassinat commis par François de La Motte, lieutenant du sieur de Montestruc, en la garnison de Mets en Lorraine..., 1607.

23. A. Saugrain, Histoire admirable et prodigieuse, d'un Pere et d'une Mere qui ont assassine leur propre fils sans le cognoistre. arrivée en la ville de Nismes en Languedoc, au mois d'Octobre dernier, 1618, Paris, 1618. 
rempli. Ces récits de meurtres ordinaires, déclenchés le plus souvent par l'appât du gain, n'avaient plus grand-chose à voir avec les attaques déclenchées pour des raisons politiques et religieuses contre les grands nobles. Ces appropriations du terme n'en rendaient pas moins ces homicides bien plus scandaleux et répréhensibles.

Tous les meurtres n'étaient cependant pas présentés comme des assassinats dans ces pamphlets. La plupart du temps, les femmes n'assassinaient pas même si les meurtrières étaient des personnages importants des canards criminels ${ }^{24}$. Dans ce genre de littérature, les meurtrières tuaient impulsivement ou alors empoisonnaient - un comportement criminel si haineux qu'il n'était pas besoin alors de terme supplémentaire pour impressionner les lecteurs. Le terme « assassinateur » était le plus souvent réservé à des hommes qui commettaient des crimes prémédités, souvent de quelqu'un de leur entourage ${ }^{25}$. Dans les canards, les victimes sont désarmées ou ne se méfient pas et le crime est alors présenté comme le résultat d'une trahison répréhensible moralement de la confiance d'autrui. Il s'agissait cependant aussi de situations dans lesquelles le meurtrier aurait pu s'exonérer de son crime, soit en échappant à la loi, soit en approchant la Chancellerie. Le canard de Philibert et Perrette décrit ainsi un meurtrier agissant sous le coup de la colère et possédé par une rage satanique mais nous pourrions tout à fait imaginer une histoire différente, dans laquelle l'accusé aurait espéré le pardon royal. Philibert n'avait-il pas été amené à croire que Perrette accepterait son offre de mariage ? Dans son esprit, ils étaient donc fiancés. Dans le pamphlet, Perrette est décrite comme obéissante et innocente, mais imaginez que Philibert ait pensé qu'elle fréquentait un autre homme. Dans une crise de jalousie, il aurait pu l'empoigner, ne pas entendre ses protestations d'innocence et la frapper, dans l'intention de lui donner une leçon et non de la tuer. Selon la loi française, un mari avait le droit de punir sa femme pour adultère, et le fait que Philibert revendiquait le statut de prétendant aurait pu renforcer sa demande de pardon. Bien des maris qui avaient tué leur femme infidèle ont obtenu une lettre de rémission sur un récit de pardon encore plus léger ${ }^{26}$.

Par définition, et parce que les accusés qui demandaient un pardon réclamaient d'être excusés, les lettres de rémission justifiaient et même exagéraient la prévalence de la violence dans la société française. Ces textes cherchaient à normaliser l'acte et à l'inscrire dans un code de l'honneur masculin largement partagé, dans lequel les insultes verbales précédaient souvent la violence physique et les querelles familiales latentes pouvaient exploser à tout moment. À la différence des canards, les récits de pardon étaient généralement désacralisés : le christianisme était rarement mentionné sauf pour dénigrer le caractère de la victime ${ }^{27}$. Au contraire, les lettres de rémission essayaient d'exonérer l'accusé en narrant les événements sur un mode accidentel, ignorant délibérément que la violence perpétrée pouvait avoir été préméditée, ce qui aurait alors entrainé aux yeux de la loi un châtiment

24. Thierry Pech, Conter le crime..., op. cit, p. 274-276. Il arrivait que des femmes sont parfois appelées assassins ; voir, par exemple Lariat, Discours de la sentence de mort donnée contre Mademoiselle Brunel, prisonniere à Grenoble, où l'on voit sa grace et commutation de peine..., Lyon, 1610.

25. Jugement notable donné à Orléans sur certain assassinat commis au pays de Vendômois, Paris, N. Le Coq, 1574, p. 9.

26. Natalie Davis, Fiction..., op. cit., p. 95 n. ; Claude Gauvard, «Entre justice et vengeance : le meurtre de Guillaume de Flavy et l'honneur des nobles dans le royaume de France au milieu du XV siècle », dans Jacques Paviot et Jacques Verger (dir.), Guerre, pouvoir et noblesse au Moyen Âge, Paris, Presses de l'Université de Paris-Sorbonne, 2000, p. 296 ; Julie Hardwick, Family business : litigation and the political economies of daily life in early modern France, Oxford, Oxford University Press, 2009, p. 183-221. Au contraire, les canards jugeaient le mari s'il tuait sa femme adultère ; voir Histoire tragique d'un gentilhomme savoyard, qui ayant trouve sa femme adultère, la fit tuer par ses deux propres enfants..., n. d.

27. Gregory Hanlon, «Les rituels de l'agression en Aquitaine au XVII ${ }^{\mathrm{e}}$ siècle », Annales. Économies, Sociétés, Civilisations, vol. 40, 1985, p. 244-268. 
complet. Les récits de pardon parvenaient à leurs fins en rappelant que la partie coupable était soit très jeune (et donc pas totalement responsable) soit, au contraire, marié et chargé de jeunes enfants (et donc important pour la communauté) ; en insistant sur le fait que la rencontre était due au hasard ou que l'importance des dommages infligés à la victime était accidentelle ; ou encore en dénigrant cette dernière comme un ennemi de longue date ou une épouse infidèle ${ }^{28}$. Ils cherchaient à minimiser l'horreur de la violence commise et de construire une narration dans laquelle le criminel pouvait être imaginé comme un individu raisonnable qui avait agi sans désir de tuer. Ce faisant, ils décrivent la violence meurtrière des hommes, en particulier des nobles, comme faisant partie de la vie ordinaire.

Les pamphlets sur les crimes font le contraire : ils fonctionnent sur le plan rhétorique comme des anti-récits de pardon focalisés sur la condamnation. Le langage des canards cherche à accentuer l'horreur du crime dans le contexte plus large de la justice sacrée : les assassins sont décrits, selon les cas, comme «ce tygre cruel $^{29}$ », « barbares », « canniballes », « beste la plus farouche du monde ${ }^{30}$ », or « un Catholique qui a abandonné son Dieu $^{31} »$. Dans les canards, les meurtriers sont invariablement des canailles et l'innocence des victimes est toujours proclamée : elles étaient aimées de leur famille ; elles étaient des femmes vertueuses ou des voisins conciliants ${ }^{32}$. Les meurtriers usaient toujours d'une force excessive : les plaies multiples, les meurtres combinés avec un viol ou une embuscade étaient très fréquents. Tout cela était inscrit dans un contexte religieux explicite : le diable était souvent à l'origine des actions des criminels ; l'intervention providentielle de Dieu conduisait parfois à l'inculpation du criminel et l'accusé redécouvrait souvent sa conscience dans les heures qui précédaient sa mort ${ }^{33}$. Bien qu'ils soient tout aussi travaillés sur le plan littéraire qu'un bon récit de pardon, nous devons considérer ensemble ces deux types de narrations si nous voulons comprendre les frontières de la violence dans la société française de l'époque moderne. Les canards qui présentaient un homicide comme un assassinat, cherchaient à condamner non seulement les professionnels stipendiés définis comme des assassins par la loi française de l'époque mais aussi une gamme plus variée d'agresseurs. En liant l'homicide avec le crime plus sérieux et plus sensationnel qu'était l'assassinat, les canards criminels ont entrepris de rendre punissables tous les homicides prémédités et ont encouragé le roi à assumer une vengeance sanctionnée par Dieu.

Les demandes désacralisées et rationnelles de pardon que l'on trouve dans les lettres de rémission et les narrations hautement confessionnalisées et articulées autour de la justice rétributive que l'on trouve dans les canards sont, en fait, les deux faces d'une même monnaie. Il y a bien sûr d'importantes différences fonctionnelles entre ces genres que nous ne pouvons ignorer : les récits de pardons étaient des documents judiciaires créés pour susciter une réponse dans le cadre d'un procès en cours alors que les canards criminels étaient publiés postérieurement à une affaire et faisaient appel au jugement du public.

28. Pour des parallèles en Angleterre, voir Peter King, Crime, justice, and discretion in England, 1740-1820, Oxford, Oxford University Press, 2000, p. 297-333.

29. N. Serpolet et B. Farge, Discours..., op. cit., p. 5.

30. Jugement notable..., op. cit., p. 10-11.

31. Meurtre exécrable arrivé aux faulxbourgs S. Marcel lez Paris, en la rue de l'Orsene, commis par Thomas Gandon, bonnetier, en la personne de sa femme, le jour Sainct Martin dernier, Paris, N. Alexandre, 1615, p. 1.

32. Discours faict sur le terrible assassinat, commis à la maison d'un gentilhomme de la Franche-Comté, par des Coquillards..., Lyon, 1605 ; Exemplaire punition..., op. cit. ; A. Saugrain, Histoire admirable..., op. cit. ; Histoire véritable d'un assassinat commis en la personne d'un jeune gentilhomme de Provence, par un jeune homme qui a esté executé en la place de Grève avec ses complices, le 19 juin 1627, Paris, C. Morlot, 1627.

33. Cruel et sanglant Assassin commis par une jeune fille âgée de vingt-un ans, paroisse de SainteMarguerite de Paris, faubourg Saint-Antoine... ; Histoire veritable..., op. cit. 
Néanmoins, les deux genres cherchent des solutions rhétoriques et pratiques face à une violence incontrôlée dans une société rendue plus sensible à ses dangers par des décennies de guerre civile et d'assassinats royaux. Tandis que les récits de pardon clamaient que l'individu pouvait être racheté et devait être réinséré dans la société humaine, les canards insistaient sur le fait qu'une telle réinsertion était impossible et que le seul espoir pour la rédemption du criminel résidait dans la grâce de Dieu obtenue sur l'échafaud.

Les canards criminels partageaient certaines caractéristiques avec un autre genre judiciaire de premier plan : le factum ou le mémoire judiciaire. Ces documents étaient commandités par les plaignants désireux de faire pression sur les juges qui enquêtaient sur une affaire, dans laquelle souvent un criminel cherchait une lettre de rémission. Les familles des victimes avaient à leur disposition un certain nombre de moyens pour influencer le processus du pardon et son rôle dans une culture de l'honneur et de la vengeance : elles pouvaient protester lorsque les récits de pardon étaient sur le point d'être enregistrés devant la cour criminelle ; elles pouvaient essayer de déplacer l'affaire devant une cour plus encline à les écouter; ou bien elles pouvaient attaquer de sang-froid le meurtrier ${ }^{34}$. Une poignée de familles de victimes choisirent pourtant l'imprimé comme un médium pour tenter de peser sur le résultat du processus judiciaire. Les premiers factums imprimés furent publiés dans le second quart du XVI ${ }^{\mathrm{e}}$ siècle, c'est-à-dire durant les mêmes décennies que les premiers canards criminels, une simultanéité qui souligne la fluidité des frontières entre les deux genres.

Les similitudes entre les canards et les mémoires judiciaires sont plus importantes que leurs différences et parfois la limite entre les deux est très difficile à tracer, en particulier en ce qui concerne leur usage du terme « assassin ». En 1579, Damoiselle Iehanne d'Amilly, veuve de Claude de Grenaise, sieur du Plessis Dechelles, en appela aux magistrats du Parlement de Paris, et au public des élites lettrées, en racontant la triste histoire de l'assassinat de son mari des mains d'un homme appelé Jean Phelipeaux, sieur de la Madeleine ${ }^{35}$. Selon le factum, un voisin avait demandé à Phelipeaux de tuer du Plessis. L'auteur du pamphlet nie explicitement que la victime ait eu le moindre conflit antérieur que ce soit avec son voisin malveillant ou bien avec son meurtrier, un démenti rédigé avec tant de véhémence que le lecteur se demande si ce n'est pas le contraire qui était vrai ${ }^{36}$. Le pamphlet prend aussi bien soin de décrire le meurtre dans des termes que les juges ne pouvaient manquer d'interpréter comme des circonstances aggravantes. Ayant été invité un soir dans la maison de son voisin, du Plessis est arrivé en toute quiétude et désarmé ; il n'avait aucune idée de ce qui l'attendait. Alors que du Plessis entrait dans la maison en plaisantant avec son hôte, le meurtrier «sans parler, sans dire mot quelconque iceluy Phelipeaux s'avança un pied dehors, sortant de derrier l'huis ou il estoit caché, \& luy donna au costé gauche de l'espee au travers du $\operatorname{corps}^{37} »$. Au cas où les juges n'auraient

34. Christiane Plessix-Buisset, Le criminel devant ses juges en Bretagne aux XVI et XVII siècles, Paris, Maloine, 1988, p. 110-112 ; Stuart Carroll, Blood..., op. cit., p. 185-206 ; Daniel Lord Smail, The consumption of justice : emotions, publicity and legal culture in Marseille, 1264-1423, Ithaca, NY, Cornell University Press, 2003.

35. Faict sommaire de l'homicide \& assassinat inhumainement \& de guet apend commis en la personne de deffunct Claude de Grenaise, vivant escuyer Sieur de Plessis Dechelles...

36. En fait, un factum publié pour le compte de Phelipeaux insista de manière emphatique sur le fait que lui et Du Plessis étaient des ennemis de longue date et que Du Plessis avait à maintes reprises ourdi des « conspirations préméditées » contre lui. Plaise à Nosseigneurs de parlement considérer et remarquer au procès d'entre Jehan de Phelipeaux,... sieur de la Magdeleine, demandeur à l'entérinement d'une lettre de rémission, contre damoiselle Jehanne d'Amilly, veuve du feu sieur du Plessis Des-Chelles, défenderesse.

37. Faict sommaire..., op. cit., p. 2. 
pas pleinement apprécié la gravité de ce crime, le factum note que « la forme du meurdre rendent ce faict odieux et punissable ${ }^{38} »$.

L'auteur explique que le pamphlet est une tentative explicite pour empêcher l'enregistrement des lettres de rémission que Phelipeaux avait déjà obtenues et dans lesquelles il avait admis avoir commis le meurtre au cours d'un « guet-apens », c'est-à-dire avec préméditation. La plaidoirie de la veuve était donc basée sur le principe légal selon lequel la préméditation devait empêcher l'attaquant d'obtenir le pardon royal. Quoique ce texte soit clairement un factum rédigé pour influencer une affaire pendante devant le parlement de Paris, sa rhétorique axée autour de l'affirmation de la vérité, son insistance sur le fait que la préméditation devait entraîner la qualification comme assassinat, sa description sans ambiguiité de la victime comme innocente, et son soutien à un châtiment physique reflètent des lieux communs que l'on trouve dans les canards.

En fait, il est tout à fait possible que les auteurs des factums aient aussi rédigé des canards. Quoiqu'ils soient le plus souvent publiés sans attribution, les historiens considèrent généralement que les mémoires judiciaires étaient écrits par des hommes de loi, qui avaient une connaissance précise du système judiciaire ${ }^{39}$. Maurice Lever a suggéré, au contraire, que les canards étaient écrits par des clercs mais il a admis lui-même que les preuves à l'appui de sa théorie étaient très minces ${ }^{40}$. Il est frustrant pour l'historien que presque tous les canards aient été publiés de manière anonyme, sans privilège, par de petits imprimeurs relativement marginaux ${ }^{41}$. Mais s'il est évident que les canards étaient lourdement chargés d'un message moral et spirituel, il l'est tout autant que leurs auteurs connaissaient à fond les détails des exécutions qu'ils décrivaient avec autant de précision. Les clercs n'avaient certainement pas le monopole de l'exaltation religieuse durant une période au cours de laquelle de nombreux laïcs étaient activement engagés dans la réforme des mœurs ou la fondation de nouveaux ordres religieux ${ }^{42}$. De plus, de nombreux historiens ont avancé que les connaissances juridiques étaient assez répandues dans une société française dont le degré de litigiosité était assez élevé et où même le menu people portait régulièrement ses querelles devant les cours de justice ${ }^{43}$. Nous pouvons donc penser que les canards étaient rédigés par la même catégorie de gens de lettres que les autres sortes de pamphlets occasionnels : des domestiques, des secrétaires de nobles, des professeurs, des clercs et des hommes de loi $^{44}$. Nous ne devons pas considérer qu'il y avait une barrière idéologique ou sociale entre les auteurs de mémoires judiciaires et ceux des canards pas plus que nous devons penser qu'ils étaient destinés à des publics complètement différents.

38. Ibid., p. 4.

39. Sarah Maza, Private lives..., op. cit., p. 1-17.

40. Maurice Lever, Canards..., op. cit., p. 14-15, 24.

41. Jean-Pierre Seguin, L'information..., op. cit., p. 14-20.

42. Barbara B. Diefendorf, From Penitence to piety : pious women and the Catholic Reformation in Paris, New York, Oxford University Press, 2004 ; Alain Tallon, La Compagnie du Saint-Sacrement, 1629-1667, Paris, Editions du Cerf, 1990.

43. Julie Hardwick, Family business..., op. cit. ; Zoë A. Schneider, The king's bench : bailiwick magistrates and local governance in Normandy, 1670-1740, Rochester, NY, University of Rochester Press, 2008 ; Jacob Melish, «Women and the courts in the control of violence between men : evidence from a Parisian neighborhood under Louis XIV », French Historical Studies, vol. 33, 2010, p. 1-31.

44. Henri-Jean Martin, Livre, pouvoir et société à Paris au XVII siècle, Genève, Droz, 1969, 2 vol., t. 1, p. 268-271; Hubert Carrier, La Presse de la Fronde (1648-1653) : les mazarinades, Genève, Droz, 1989, 2 vol., t. 2, p. 3-40, 77-86, 105-112 ; Hélène Duccini, Faire voir, faire croire : l'opinion publique sous Louis XIII, Seyssel, Champ Vallon, 2003, p. 49-54 ; Jeffrey K. Sawyer, Printed Poison : Pamphlet Propaganda, Faction Politics, and the Public Sphere in Early Seventeenth-Century France, Berkeley, University of California Press, 1990, p. 52-54. 
Le goût des canards pour le surnaturel et l'extraordinaire semble les différencier des mémoires judiciaires mais il s'agit d'une distinction plus apparente que réelle car la plupart des récits criminels étaient moralisants, mélodramatiques et sanglants. En fait, la principale différence entre les deux genres est que les canards racontent en général un crime et sa punition comme un fait accompli alors que les mémoires judiciaires sont publiés pendant le déroulement d'un procès. Les canards, comme les mémoires judiciaires, sont publiés pour un public alphabétisé dont ils espèrent gagner la sympathie pour une cause que l'auteur considère comme juste. Quoique l'idée abstraite d'un tribunal de l'opinion publique ne soit jamais exprimée dans ces textes, leur existence même indique qu'un discours public sur l'efficacité de la justice criminelle existait en France dès la fin du XVI $\mathrm{I}^{\mathrm{e}}$ siècle $^{45}$.

L'efficacité de cette littérature pamphlétaire peut être testée grâce à l'analyse d'un de ces textes, parfois considéré comme un canard, qui s'appuie sur le récit d'une intervention surnaturelle et miraculeuse pour influencer les pratiques judiciaires du Parlement de Paris. Dans un long pamphlet, Martial Deschamps, un docteur et humaniste bordelais, essaya, en effet, d'obtenir la condamnation des auteurs d'un assassinat, son propre assassinat, bien qu'il ait survécu pour raconter son histoire ${ }^{46}$. Les malheurs de Deschamps découlent de sa décision de défendre les droits d'une veuve et de sa fille sur un domaine ${ }^{47}$. La veuve, Jeanne de La Chassaigne, était la femme d'un ancien conseiller au Parlement de Bordeaux, un Huguenot notoire, qui avait été tué durant le massacre de la Saint-Barthélemy en 1572, ce qui peut d'ailleurs expliquer pourquoi Deschamps insiste autant dans le texte sur sa propre foi catholique ${ }^{48}$. Après la mort de son mari, sa veuve entrepris de récupérer une propriété à elle, qui avait été confisquée par un noble, Gaspard Foucault, sieur de Beaupré, lui aussi un Huguenot, qui était un ambitieux potentat local à la réputation douteuse ${ }^{49}$. La propriété contestée avait été vendue en 1512 par un ancêtre de Beaupré à l'arrière-grand-père de

45. Beaucoup d'historiens ont affirmé, en suivant Jürgen Habermas, qu'une sphère publique sécularisée a commencé à émerger au milieu du XVII ${ }^{\mathrm{e}}$ siècle et qu'elle a pleinement mûrie simplement un siècle plus tard. Voir Sarah Maza, Private lives..., op. cit. ; Hélène Merlin, Public et littérature en France au XVII siècle, Paris, Belles Lettres, 1994 ; Alain Viala, Naissance de l'écrivain : sociologie de la littérature à l'âge classique, Paris, Editions de Minuit, 1985 ; Keith Michael Baker, Inventing the French Revolution : Essays on French Political Culture in the Eighteenth Century, Cambridge, Cambridge University Press, 1990 ; Christian Jouhaud, « Les libelles en France dans le premier XVII ${ }^{\mathrm{e}}$ siècle : lecteurs, auteurs, commanditaires, historiens », XVII ${ }^{e}$ Siècle, vol. 49, 1997, p. 203-217. Néanmoins, un débat public existait sans nul doute dans l'Angleterre et la France de la fin $\mathrm{du} \mathrm{XVI} \mathrm{e}^{\mathrm{e}}$ siècle à propos de l'obligation du monarque de prendre en compte le bien public ou de s'attendre à une rébellion, et il transparaît dans les pamphlets, les traités et le théâtre. Voir Sara Beam, Laughing Matters : Farce and the Making of Absolutism in France, Ithaca, NY, Cornell University Press, 2007, p. 44-76 ; James B. Collins, « Noble Political Ideology and the Estates General of Orleans and Pontoise : French Republicanism », Historical Reflections/Reflexions historiques, vol. 27, 2001, p. 219-240; Denis Pallier, Recherches sur l'imprimerie à Paris pendant la Ligue (1585-1594), Genève, Droz, 1975, p. 42-51 ; Jeffrey Sawyer, Printed Poison..., op. cit., p. $67-$ 83 ; Hélène Duccini, Faire voir, faire croire..., op. cit., p. 58-69 ; Peter Lake and Steve Pincus, « Rethinking the public sphere in early modern England », Journal of British Studies, vol. 45, 2006, p. 270-292.

46. Originaire de Limoges, Deschamps était l'ami d'humanistes comme Jean Dorat et Élie Vinet à Bordeaux dans les années 1570. Voir Camille Jouhanneaud, Jeanne d'Albret et les Limousins, Limoges, Ducourtieux, 1897, p. 10 ; J. de La Ville de Mirmont, « Jean Dorat et Élie Vinet », Revue historique de Bordeaux et du Département de la Gironde, vol. 3, 1910, p. 377-382.

47. Histoire tragique et miraculeuse d'un vol et assassinat commis au païs de Berri, en la personne M. Martial Deschamps Medecin de l'Université de Paris, \& ordinaire de la Maison \& Ville De Bourdeaux..., Paris, Iehan Bienné, 1576.

48. Sara Beam, Laughing matters..., op. cit., p. 36.

49. Gabriel O'Gilvy, Nobiliaire de Guienne et de Gascogne : revue des familles d'ancienne chevalerie ou anoblies de ces provinces antérieures à 1789, avec leurs généalogies et leurs armes, Bordeaux, Gounouilhou, 1856, vol. 1, p. 396 ; L. de Corbier, «Le Comté de Dognon en la Marche et ses seigneurs », Mémoires de la Société des Sciences Naturelles et Archéologiques de la Creuse, $2^{\text {nd }}$ sér., vol. 15, 1905, p. 638. 
Jeanne. Il semble que Beaupré ait profité de l'instabilité de la situation confessionnelle et militaire du Limousin pour récupérer son patrimoine. La Chassaigne a essayé de régler le litige par l'intermédiaire du Parlement mais, en 1576, elle n'avait toujours pas recouvré sa propriété.

Selon son récit, Deschamps, le champion de la veuve, essaya de résoudre la dispute en se rendant dans la propriété contestée, à Chastelleux, au nord de Limoges. C'était une entreprise audacieuse car Beaupré était réputé pour les violents excès de ses campagnes militaires et il opérait dans la région à cette époque. Sur la route, Deschamps et son compagnon de voyage, furent attaqués par trois hommes qui déclarèrent ouvertement être liés à Beaupré. Les trois hommes s'emparèrent de l'argent de Deschamps ainsi que du titre de propriété de Chastelleux et affirmèrent qu'ils allaient l'emmener dans un château voisin. Au lieu de cela, ils lièrent les mains et les pieds de Deschamps et de son compagnon, et ils les abandonnèrent dans un marécage au milieu de la forêt, les laissant pour morts. Deschamps insiste longuement sur le fait que seule la main de Dieu pouvait les sauver, lui et son compagnon, d'une mort certaine et il s'étend tout aussi longuement sur l'impossibilité matérielle qu'ils aient pu s'extirper eux-mêmes de ce marais sans une intervention divine. Ils étaient donc en train de se noyer quand Deschamps fit une expérience corporelle extraordinaire liée à ses prières ferventes. Les deux hommes se trouvèrent tout à coup debout ; son compagnon réussit à mordre dans les cordes qui liaient leurs mains ; et ils purent gagner en titubant un sol plus ferme. L'intervention divine qu'il raconte, affirme donc la justesse de leur cause : elle est inscrite dans une vision binaire d'un monde divisé entre le bien et le mal, qui est tout à fait familière aux lecteurs des canards.

En dépit du fait que son pamphlet se lit comme un canard, la plaidoirie de Deschamps fonctionne rhétoriquement comme un factum. Apprenant que Deschamps avait survécu à l'épreuve, Beaupré se rendit à Paris pour demander le pardon royal. Le pamphlet de Deschamps dessine un récit alternatif qui insiste sur le fait que le crime commis l'assassinat de gens pris au dépourvu - n'était pas sujet au droit de grâce. Et il remporta l'affaire. Selon un arrêt publié en 1576, la même année que le pamphlet, le Parlement de Paris condamna Beaupré à être décapité et deux des hommes qui avaient attaqué Deschamps, à être démembrés et à mourir sur la roue. Le troisième conjuré, un clerc, devait être châtié par l'évêque de Poitiers. De plus, tous les biens du sieur de Beaupré devaient être confisqués, des réparations devaient être payées et la propriété contestée rendue à Jeanne de La Chassaigne $^{50}$. Quoique l'arrêt ne puisse pas être pleinement appliqué, puisque Beaupré resta en liberté et rejoignit les forces d'Henri de Navarre dans les années qui suivirent, il l'empêcha néanmoins de faire enregistrer ses lettres de rémission. En outre, la veuve regagna le contrôle de la propriété de Chastelleux ${ }^{51}$. Le pamphlet et l'arrêt qui s'en suivit indiquent donc la manière dont le mot assassinat pouvait renforcer l'affirmation par le plaignant qu'un châtiment et non un pardon était le légitime cours de la justice. Quoique le texte ait été publié seulement après la conclusion du procès, il est tout à fait plausible, compte tenu du fait que Deschamps était un humaniste très respecté, qu'il ait circulé sous une forme manuscrite avant la sentence. En s'appuyant sur des récits convaincants et familiers, lourdement chargés de morale chrétienne, les pamphlets du genre de celui

50. Dans la série X2a des Archives nationales, les arrêts n'existent plus pour les années entre 1571 et 1579. Néanmoins, Martial Deschamps était un humaniste connu et respecté, et le pamphlet a été publié avec un privilège royal. Ces deux faits me font donc penser que les indications du pamphlet sur le contenu de l'arrêt sont véridiques. Pour une lecture différente de ce pamphlet, comme un canard, voir Natalie Davis, Fiction..., op. cit., p. 65. p. 562 .

51. Gabriel Ogilby, Nobiliaire..., op. cit., p. 396-399 ; L. de Corbier, « Le Comté de Dognon... », op. cit., 
de Deschamps cherchaient donc à persuader de la justice de la cause des victimes et à influencer ses lecteurs quant à la nécessité de la justice rétributive pour la société française.

Les premiers mémoires judiciaires, comme celui de Deschamps, illustrent donc les nombreuses ressemblances entre les plaidoiries judiciaires formelles et les canards criminels de la fin du XVI ${ }^{\mathrm{e}}$ siècle. Les deux genres dénonçaient le meurtrier en invoquant le code de l'honneur, la morale chrétienne aussi bien que la dévotion pour le roi et pour Dieu. La justice était représentée comme une défense à la fois de l'ordre politique et de la loi naturelle ce qui garantissait la justesse d'une rétribution violente contre les criminels avérés. Ces textes ne pouvaient naturellement pas changer le fait que la justice royale n'était pas assez forte, à la fin du XVI ${ }^{\mathrm{e}}$ siècle, pour limiter la prolifération des actes de vengeance privée, mais ils n'en étaient pas moins des prises de position publiques qui insistaient sur la valeur morale et la nécessité pratique d'une justice royale forte désireuse de tirer vengeance de ses ennemis.

Le langage de l'assassinat n'existait cependant pas seulement dans les pamphlets de la fin du XVI ${ }^{\mathrm{e}}$ et du début du XVII ${ }^{\mathrm{e}}$ siècle. Il se trouvait également dans les archives, non pas dans les lettres de rémission, naturellement, dans les arrêts rédigés par les magistrats des parlements, où appeler un crime un assassinat était souvent une tactique pratique pour les plaignants. Les affaires de meurtres jugées par les cours étaient le plus souvent appelées des homicides mais, en raison du fait que beaucoup d'affaires criminelles étaient à cette époque suscitées par des parties civiles qui cherchaient des compensations financières, les accusateurs qui initiaient (et finançaient) une affaire, avaient toutes les raisons d'insister sur la dépravation morale de l'accusé ${ }^{52}$. En conséquence, la caractérisation d'une attaque ou d'un meurtre comme un « assassinat » devint une stratégie couramment utilisée devant les parlements dès les années 1570, sans doute parce que les avocats ont commencé à utiliser ce langage lorsqu'ils plaidaient devant les cours. Bonnaventure de la Tousche, par exemple, a ouvert une procédure devant le sénéchal d'Anvaye et, plus tard, devant le parlement de Bretagne, en 1577, pour «l'assassignat commis en sa personne par ung homme masqué », nommé dans le texte comme étant Guy d'Avangour, sieur de Vey ${ }^{53}$. La tentative de la Tousche de faire reconnaître l'attaque menée contre lui comme un assassinat a finalement échoué, comme cela était souvent le cas. Les conseillers semblent avoir été souvent réticents à accepter l'interprétation très large du terme d'assassin qui était celle des avocats. En revanche, deux bergers, Jehan Dardenne et Anthoine Le Sellier, accusés d'avoir commis un « homicide et assassinat » contre Crespin Dardenne, ont été, en mars 1584, traités bien plus sévèrement. Le Parlement de Paris a décidé de les faire torturer tous les deux, et, sur la base de leur confession, a conclu que l'un d'entre eux, Jehan Dardenne, devait être pendu $^{54}$. Le fait que les conseillers reconnaissaient parfois que l'usage polémique du terme « assassinat », qui caractérisait les avocats, avait une portée légale suggère à quel point il s'était répandu à la fin du XVI $\mathrm{I}^{\mathrm{e}}$ siècle aussi bien dans les milieux judiciaires que dans les pamphlets. Loin d'être contradictoires avec les récits produits dans les pamphlets, les tactiques des plaignants révèlent le même désir d'imprimer un sens moral aux procédures judiciaires et de chercher à résoudre un conflit par une condamnation plutôt que par un pardon.

52. Christine Plessix-Buisset, Le criminel..., op. cit., p. 88.

53. AN, X2a 144, f. 262r, 22 mars 1584.

54. AN, X2a 144, f. 169r-v, 8 mars 1584. Voir aussi le cas de Paul de Vallerin, qui a été torturé, et qui, ayant refusé d'avouer, a été banni et frappe d'une amende de cent écus pour « assassinat ». AN, X2a 147, f. 131v-132r, 24 mai 1585 ; AN, X2b 1330, 23 mai 1585. 
En définitive, ce nouvel usage du terme « assassinat », dans les cours de justice aussi bien que dans les canards, a aidé à transformer la conception française du meurtre avec préméditation. À la fin du XVII ${ }^{\mathrm{e}}$ siècle, l'assassinat avait, en effet, évolué d'un sens restreint, celui d'une action menée par un meurtrier à gages, vers un sens plus proche de celui en usage actuellement. Dans le code criminel de 1670, l'assassinat est présenté comme un crime proche du duel, un privilège traditionnel de la noblesse. L'assassinat et le duel sont alors, tous les deux, définis comme des homicides irrémissibles, et la distinction fondamentale entre les deux, le paiement de l'assassin, était donc brouillée car les deux crimes apparaissaient odieux aux yeux de la loi ${ }^{55}$. L'édition de 1694 du dictionnaire de l'Académie française pousse cette évolution encore plus loin en définissant un assassin comme tout « meurtrier de guet à pens, soit en trahison, soit avec advantage ${ }^{56}$. L'usage polémique des canards de la fin du XVI ${ }^{\mathrm{e}}$ siècle, qui cherchaient à accentuer la sympathie pour la victime, était donc devenu, un siècle après, une partie de la définition normale d'un meurtrier. Ce glissement linguistique caractérise une société dans laquelle la violence meurtrière des hommes, en dehors des lieux sanctionnés par le pouvoir, en particulier les champs de bataille, était en voie de diminution ${ }^{57}$.

Ces continuités entre les manœuvres des cours de justice et les narrations de bien des canards criminels démontrent que ces documents méritent une attention plus soutenue des historiens qui cherchent à analyser la complexité des discours publics sur la violence au tournant des $\mathrm{XVI}^{\mathrm{e}}$ et $\mathrm{XVII}^{\mathrm{e}}$ siècles. Les bagarres de rue ou bien les violences conjugales n'étaient pas considérées comme des crimes sensationnels à cette époque et ils sont donc absents des canards. Au contraire, le meurtre de sang-froid d'une victime confiante dépassait ce que les auteurs de canards pouvaient accepter ; certains meurtres, avançaientils, étaient si haineux qu'ils se situaient au-delà de la pitié, et qu'ils relevaient seulement du jugement du roi et de Dieu. Dans leurs efforts pour divertir, pour choquer, pour moraliser et pour influencer le monde, les canards criminels légitimaient ainsi les châtiments violents mis en place par les officiers du roi à cette période. Loin d'encourager la clémence, une tendance démontrable des cours de justice françaises à partir de 1650, les pamphlets qui définissaient un meurtre comme un assassinant incitaient la justice publique à être vigilante et même à étendre son emprise sur tous les sujets du roi, y compris les nobles, jusqu'alors habitués à résoudre leurs différends par la violence et à obtenir le pardon du roi.

UNIVERSITY OF VICTORIA

55. Ordonnance criminelle du mois d'août 1670, titre XVI, article 4, mis en ligne le 17 août 2010. URL : http://ledroitcriminel.free.fr/la_legislation_criminelle/anciens_textes/ordonnance_criminelle_de_1670. htm. Consulté le 15 septembre 2010.

56. Dictionnaire de l'Académie Française, première édition, Paris, Veuve de Jean Baptiste Coignard, 1694, p. 60, mis en ligne le 28 mai 1997 par le projet ARTFL. URL: http://portail.atilf.fr/cgi-bin/getobject_?p.0:78./ var/artfla/dicos/ACAD_1694/IMAGE/. Consulté le 15 septembre 2010.

57. François Billacois, Le duel dans la société française des XVI ${ }^{e}$-XVII ${ }^{e}$ siècles, Paris, Éditions de l'École des Hautes Études en Sciences Sociales, 1986 ; Julius R. Ruff, Violence in early modern Europe, 1500-1800, Cambridge, Cambridge University Press, 2001. 